Ann. Abeille, I962, 5 (2), I45-I53.

\title{
UN APPAREIL POUR LE CALCUL RAPIDE DES SURFACES DE COUVAIN DANS LES RUCHES
}

\author{
J. RRESNAYE \\ Station expérimentale d'Apiculture, \\ Contre de Recherihes agronomiques du Sud-Est, Montfavet (Vaucluse).
}

SOMMAIRE

Description d'un appareil de manipulation simple, permettant d'évaluer rapidement l'importance du couvain dans les colmies d'Abeilles. Cette rapidité permet d'utiliser le critère "surface dı couvain " dans des expériences réunissant de nombreuses ruches. Il se compose essentiellement d'un abaque fournissint directement la valeur d'une surface en fonction de coordonnées indiquées par deux réglettes mobiles. Cet abą̧ue est enroulé sur un cyclindre mobile autour de son axe. La précision de la méthode ( \pm I 3 p. Ios d'erreur moyeme) est satisfaisante en expérimentation apicole.

\section{INTRODUC'TION}

L'appréciation de l'importance des surfaces de couvain dans les ruches à différentes époques de l'année ou dans certaines conditions expérimentales est d'une importance primordiale dans les recherches sur 1'Abeille. Nous avons étudié précédemment (FRESNAYE et LENSKV, I96I) différentes méthodes utilisables pour des expériences réunissant un grand nombre de colonies et nous avons comparé leur précision et le temps nécessaires à leur application. Cette étude nous a permis de constater que les méthodes très précises (moins de \pm Io p. Ioo d'erreur moyenne) étaient très lentes et que les méthodes très rapides étaient d'une précision insuffisante, (plus de \pm 20 p. Ioo d'erreur moyenne). Elle nous a également montré que la méthode du calcul de la surface de l'ellipse de couvain est somme toute la plus satisfaisante puisqu'elle ne comporte que \pm I3 p. Ioo d'erreur moyenne, tout en étant l'une des plus rapides, bien que le temps nécessaire pour effectuer les calculs soit quatre fois plus important que le temps requis pour les mesures. Ce travail préliminaire m'a amené à envisager de supprimer les calculs numériques en utilisant un appareil qui, dans sa concepttion, rappelle une règle à calcul. 


\section{DESCRIPTION I'T UTILISATION DE L'APPAREIL,}

On sait que la surface de l'ellipse est donnéc par la formule :

$$
s=\frac{1}{2} \times \frac{a}{2} \times \pi
$$

dans laquelle d et a sont les axes.

l.appareil que nous avons conçu est dest iné à applicuer directement cet te formule clans le calcul des surfaces de couvain. Il se compose essentiellement des parties suivantes:

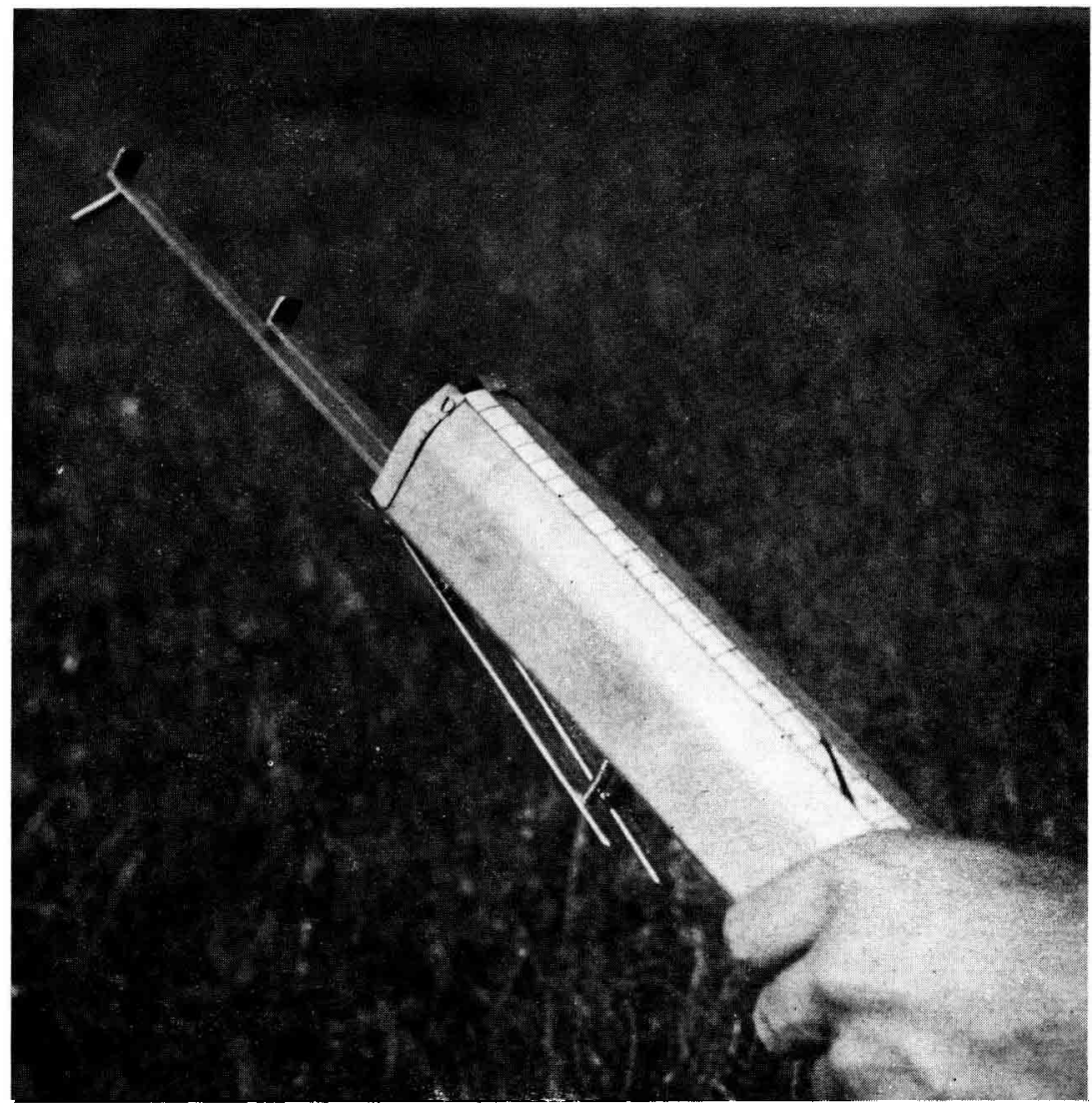

Fu. I. - Asped exterieur de l'uppareil. On voit l'abaque lans l'onterture lu boitier, les réglettes, les leviers des treins

$1^{n}$ Un cylindre mobile antour de som axe.

$2^{\circ}$ Un abaque enroulé sur le cylindre el qui représente une famille de courbes correspondant aux surfaces d'une série d'ellipses.

$3^{\text {o }}$ Deux réglettes mobiles servant à mesurer les axes des ellipses.

D) u point de vue mécanique notre appareil comporte un certain nombre de pièces dont les principales sont :

a) Un boitier métallique en tôle nince de $370 \mathrm{~mm}$ de longueur, $45 \mathrm{~mm}$ de largeur et $50 \mathrm{~mm}$ de hauteur. Ces cotes ont été adoptées pour cue l'appareil soit tenu facilement en main sans provoquer de fatigue, même après plusieurs heures d'utilisation. P'our la même raison le dessus du boîtier est arrondi et épouse la forme de la paume. Une ouverture pratiquée sur toute la longueur de l'appareil 
permet la lecture de l'abaque tandis que d'autres ouvertures permettent le passage des tiges coulissantes et des freins.

b) Une première réglelte qui porte un index et coulisse à frottement doux dans une rainure pratiquée à l'intérieur du boîtier. Elle sert à mesurer le grand axe des ellipses.

c) Une seconde réglette qui porte un goujon, lequel, en glissant dans une rainure hélicoïdale du cylindre, fait tourner celui-ci sur son axe. Flle sert ì mesurer le petit axe des ellipses.

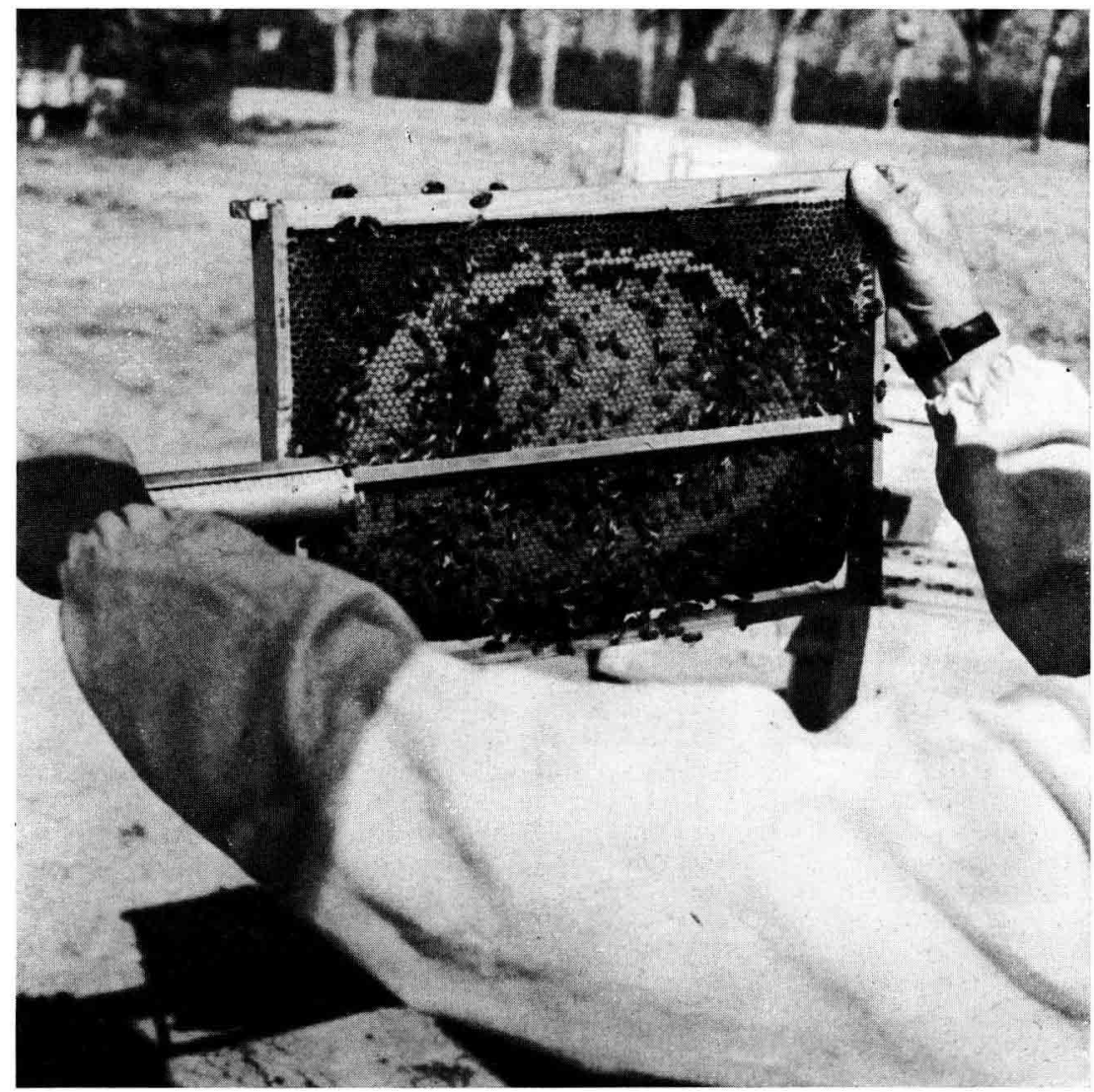

FIG. 2. - $\mathrm{I}^{\mathrm{er}}$ lemps -.. Mesure du grand axe de l'ellipse de couvain

Lorsque l'appareil est au zéro les deux réglettes dépassent du boîtier de $60 \mathrm{~mm}$, ceci afin de compenser la zone dont l'abaque est amputé (voir figure 4). Une tige ronde de $20 \mathrm{~mm}$ de long est fixée a $45^{\circ}$ vers l'extérieur à l'extrémité de chacune des réglettes. Les deux tiges forment dosc un angle de $90^{\circ}$, ce qui facilite la mesure des deux axes d'une ellipse (voir utilisation).

d) Un frein placé sur chaque réglette permet l'immobilisation au point voulu. Il est const it ué par un levier appuyant un tampon de caoutchouc sur la réglette sous la pression d'un ressort (fig. I).

e) Un cylindre creux, de $37 \mathrm{~mm}$ de diamètre et $360 \mathrm{~mm}$ de longueur et dont l'axe tourne dans deux cuvettes fixées au boitier. Une rainure hélicoïdale est forée sur une partie de la longueur et convertit en mouvement circulaire, le mouvement rectiligne du goujon fixé à la réglette ainsi qu'il est indicuué en c). 
TABLEAU I

Surface des ellipse's de I 2 à 5 points et valeur de $\frac{A}{2} \times \frac{a}{2}\left(\right.$ ou $\left.\frac{s}{\pi}\right)$

\begin{tabular}{|c|c|c|c|c|c|c|c|}
\hline $\begin{array}{l}\text { Surfaces } \\
\left(\text { cu cmi }{ }^{2}\right) \text {. } \\
\text { Valeurs } \\
\text { ext rêmes }\end{array}$ & $\begin{array}{c}\text { Surfaces } \\
(\text { en cm²). } \\
\text { Valcurs } \\
\text { moyemes }\end{array}$ & $\begin{array}{c}\text { Nombre } \\
\text { de points } \\
\text { correspond. } \\
\text { à la valem } \\
\text { movenne }\end{array}$ & $\begin{array}{c}\text { Valeur } \\
\text { correspondte } \\
\text { (e } \frac{A}{2} \times \frac{a}{2} \\
\left(\text { ou } \frac{\mathrm{S}}{\pi}\right) \\
\text { des extrimes* }\end{array}$ & $\begin{array}{c}\text { Surfaces } \\
\left(\text { cu cm }{ }^{2}\right) . \\
\text { Valeurs } \\
\text { extrenes }\end{array}$ & $\begin{array}{c}\text { Surfaces } \\
\left.\text { (en } \mathrm{cm}^{2}\right) \text {. } \\
\text { Valenrs } \\
\text { movenues }\end{array}$ & $\begin{array}{c}\text { Nombre } \\
\text { de points } \\
\text { correspond. } \\
\text { à la villeur } \\
\text { moveme }\end{array}$ & 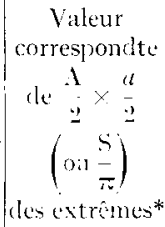 \\
\hline$*$ & & | & & $*$ & & i & \\
\hline $20-(i)$ & 40 & 1 & $6,: \because 6 ;$ & (3)-50 & $5(i)$ & $1 t$ & 171,97 \\
\hline $60-100$ & xil & $\therefore$ & 19,10 & EN1)-1;00 & 600 & $1 ;$ & $\mid$ \\
\hline $100-140$ & $1: 0$ & ?3 & 131, & $1 ; 20-6 ; 60$ & $(0 ; 0)$ & 16 & 1917,45 \\
\hline $1 / 40-180$ & 16,0 & 't & A & C,60-700 & 680 & 17 & $290,1 !$ \\
\hline $180-2=0$ & $\because 00$ & 5 & $57, \because 2$ & $700-7 \div 0$ & 7211 & $1 n$ & $2,-3,92$ \\
\hline $220-260$ & 210 & i) & 70,06 & $740-780$ & $7(61)$ & $1 ! 1$ & $2: 35,166$ \\
\hline $260-300$ & 280 & 7 & $\therefore 2,80$ & T(1)-8:0 & s(ii) & 211 & ב4: \\
\hline $300-360$ & $: \because 20$ & 8 & 95,14 & $8: 0-81 ; 0$ & אill & 21 & $=261.111$ \\
\hline $310-380$ & 360 & 9 & 108,24 & $860-9600$ & (5x) & 22 & $273, \mathrm{~s}$ \\
\hline$: 380-1290$ & 400 & 10 & 121,01 & (9)(1)-9) & $9=0$ & $2 ;$ & 286,60 \\
\hline$\{=0-\{0,1)$ & (1911 & 11 & 133.75 & $910-930$ & ario & $\because 4$ & 2999,36 \\
\hline$\therefore 60-500$ & 你保 & 12 & $1,6,19$ & $980-10 \div 0$ & $10(101)$ & $\because 5$ & $: 12.111$ \\
\hline $500-510$ & $5: 0$ & 13 & $159,2,: 3$ & 1020 & & & ' \\
\hline
\end{tabular}

f) L'abaque (fig. 4) est enroulé autour du cylindre. Il permet de lire directement les surfaces déduites des mesures effectuées au moyen des réglettes. Sa lecture se fait en points de valcur arbitraire correspondant a fo $\mathrm{cm}^{2}$. Dans cés conditions te cadre de ruche "I adant " vaut 25 points par face de cadre (ces carlres, correctement const ruits, représentent environ $1000 \mathrm{~cm}^{2}$ de celluler par face). Ies cires gaufrées que nous ut ilisons présentent 750 cellules au dmo module très courant en apiculture pratique ${ }^{1}$ ) soit 375 cellules par dn ${ }^{2}$ et par face. Aver notre appareil, le point correspond dont: exactement ì 50 cellules. Un rapide calcul mental permet de comertir í volonté les pointsen $\mathrm{cm}^{2}$ ina en nombre de cellules, ceci selon les iresoins. Limité de surface choisie, autrement dit le point, permet une lecture facile de l'abague tout en maintenant une précision expérinentale satisfaisante. Le tableau i donne la surface des ellipses correspondant a un nombre de points allant de 0 it 25 ainsi

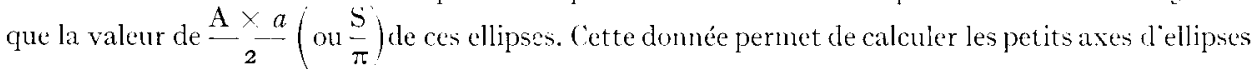
dont la surface et le grand axe sont comnus (tableau 2). Sur l'abactue, les points sont inscrits entre des courbes calculées et tracées avec précision, l'inscription est répétée régulièrement afin de permettre la lecture dans toutes les positions du cylindre dont une faible partic seulement apparait dans la fenêtre du boîtier.

Le tracé de l'abauque a été étudié pour pernettre l'utilisation maximum de la surface du cylindre. C'est ainsi culune partie de la surface de l'abayue correspondant ia des ellipses hautement improbables dans les bandes I, 2 et 3 est récupérée au profit des bandes plus fréquemment utilisées. De ce fait la circonférence du cylindre a pu être réduite sans nuire à la précision de l'appareil.

Pour réduire l'encombrement et alléger l'appareil, l'abaçue a été amputé des $60 \mathrm{~mm}$ qui constit uent son début. Cette partie correspond à des surfaces d'une valeur inférieure à i point, négligeables dans les calculs.

La ligne $\mathrm{AB}$, tracée approximativement en diagonale sur le graphique a plusieurs utilisations. lille est suivie par l'index lorsque la surface mesurée est un cercle. Lors de la construction de lappareil on fixe l'abaque de telle sorte que le point A se trouve en regard de l'index. Fn sortant progressivement les deux réglettes tris exactement ensemble l'index doit suivre la ligne AB ce qui garantit la mise en place correcte de l'abaque. D'autre part, la ligne AB est parallèle à la rainure hélicoìdale, çui se trouve diamétralement opposée et peut donc servir à son traçage. On doit toujours mesurer le grand axe de l'ellipse avec la réglette| qui porte l'index et le petit axe avec la réglette qui fait tourner le cylindre ; ainsi l'index se trouve toujours dans la partie droite inférieure de l'abaque. L'inversion

(1) Rappelons que le nombre de cellules au dm s'entend toujours pour les deux faces d'un rayon. 
des nesures est sans importance daus le cas d'ellipses dont les axes sont peu différents, mais peut être cause d'erreurs dans le cas contraire en faisant entrer l'index dans les bandes I et 2 alors que la surface à mesurer correspond à 8 ou 12 points. Aprés quelques essais on s'apercevrait d'ailleurs facilement de ces erreurs grossières. Une ligne double soulignée en rouge sépare la partie haute et la partic basse de l'abayue après l'enroulement de celle-ci : les mesures prises avec la réglet te qui porte l'index sont a échelle I alors que les mesures prises avec la réglette qui fait pivoter le cylindre sont à l'échelle I / 2 sur l'abaque en raison du faible dianèt re du cylindre (37 mun de diamèt re et I i 7 num de circonférence). Ia partie récupérée sur les bandes $\mathbf{I}_{-2-3}$ no:k permet de porter la hauteur de l'abacpue à $140 \mathrm{~mm}$ et de mesurer ainsi des ellipses dont le pet it axe a une longueur maximum de $280 \mathrm{~mm}$ et le grand ixe $420 \mathrm{~mm}$. (I aibague reproduil lig. I permed de mesurer des grands axes de 4 to mme).

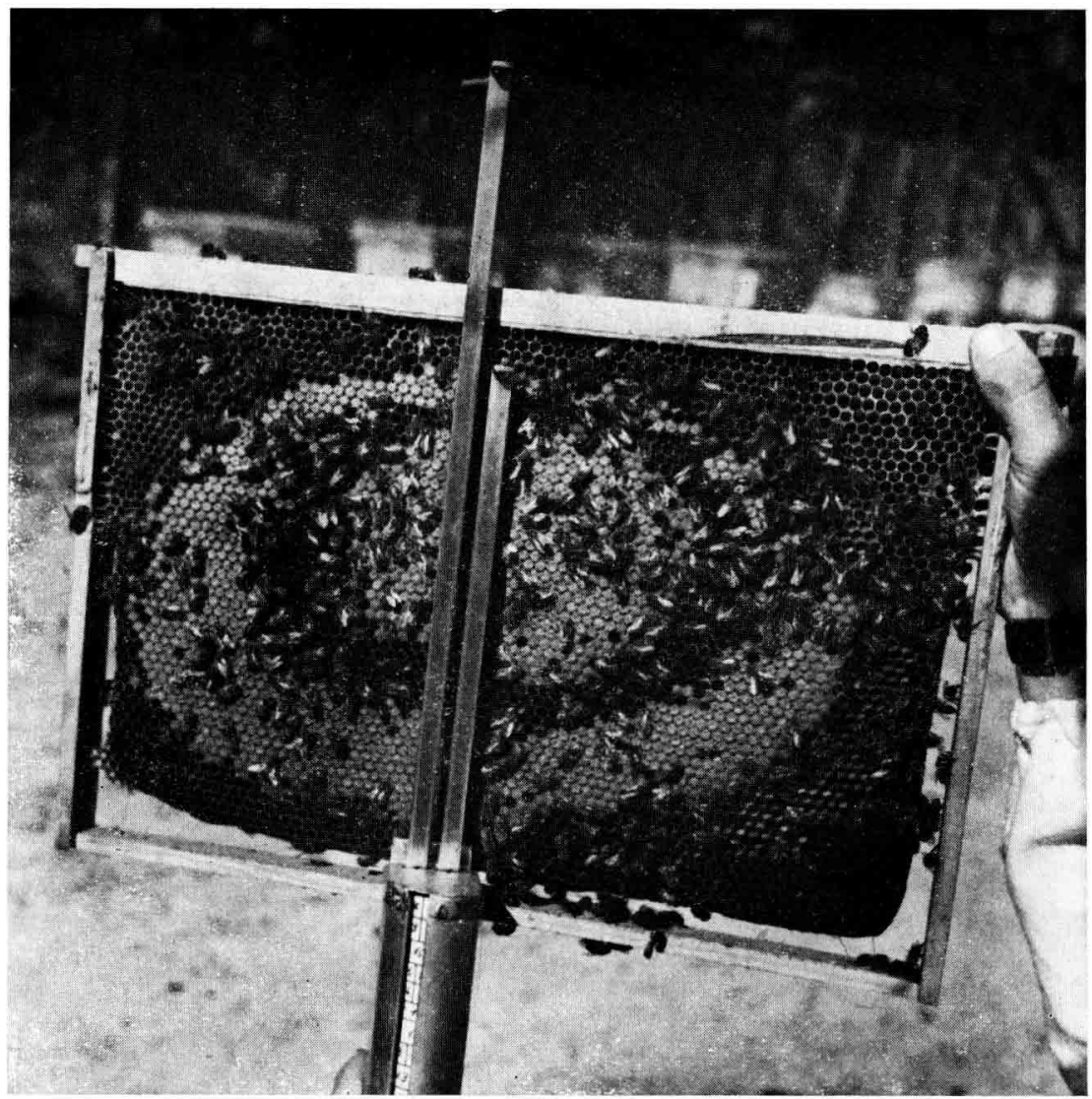

IIr. 3. - $2^{e}$ temps - Mesure du petil axe de l'ellipse de comrain.

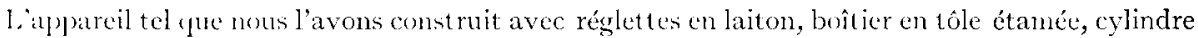
en acier doux, pèse cnviron I 300 grammes.

I appareil se tient d'une main et par son milieu. I ans cette position les leviers des freins prennent place nat urellement sous deux doigts. (es leviers sont de longueurs différentes et doivent ètre interververtis selon que l'utilisateur tient l'appareil de l'une on de l'autre main.

I) méx méthodes d'utilisation peuvent être envisagées.

a) . Withode d'utilisation da deux personnes. C'est la plus pratique et celle fui donne les nueilleurs résultats. L'un des expérimentateurs visite normalement la ruche et présente successivenent les cadres au second expérinentateur qui prend les mesures en tenant l'appareil cl'une main et les réglettes atlemativement de l'autre main. Un repère à lavant du boîtier, est présenté à une extrémité du grand axe de l'ellipse. Ia réglette portant l'index est tirée jusqu'à ce que la tige ronde se trouve à l'autre extrémité de cet axe; on tient l'appareil de façon à ce que cette tige ronde soit perpendicu- 


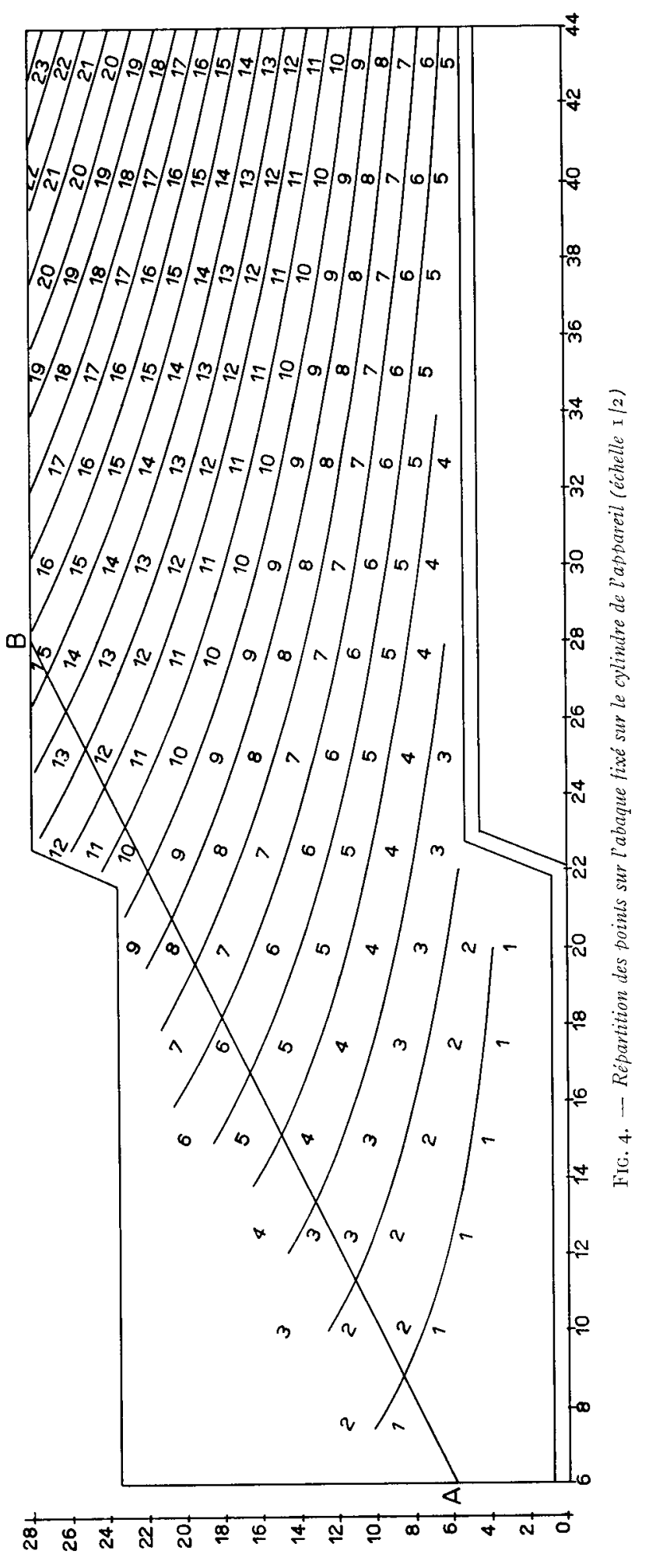




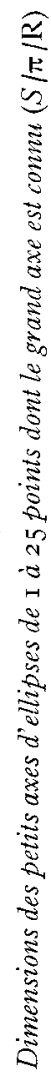

Petit axe des ellipses (en $\mathrm{cm}$ )

$\stackrel{g}{8}$

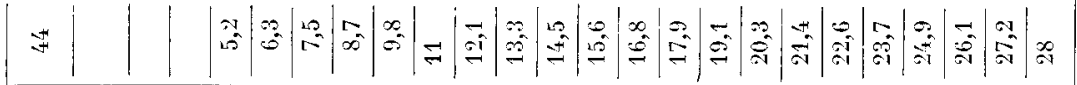

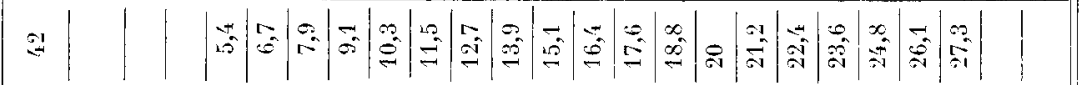

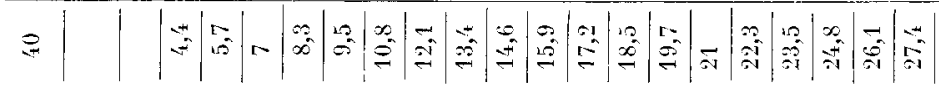

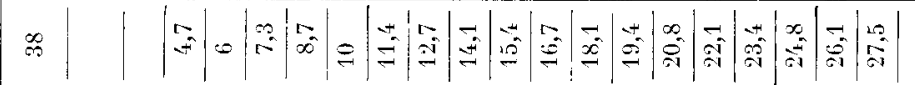

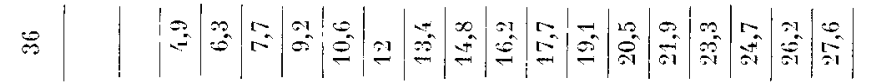

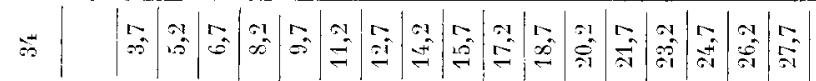

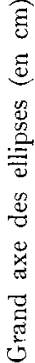

舫

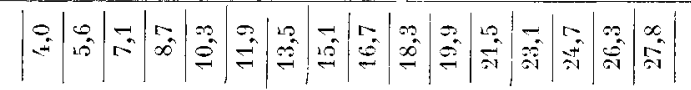

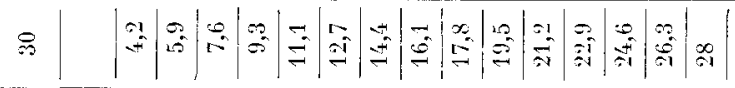

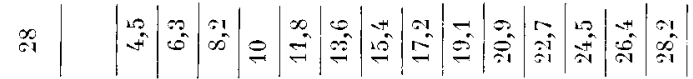

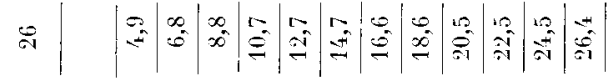

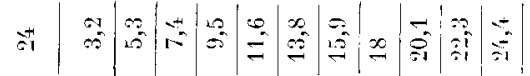

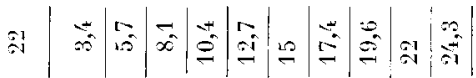

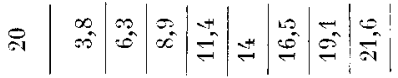

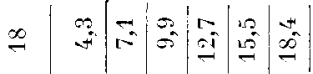

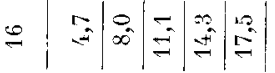

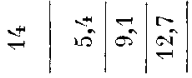

$\because\left|\begin{array}{l|l}\infty & 0 \\ 0 & 0\end{array}\right|$

$\because \stackrel{6}{5}$

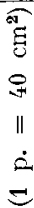

需

॥ 
laire à la surface du cadre. On procède de la même manière avec le petit axe de l'ellipse en inclinant l'appareil de l'autre côté pour que la tige ronde de la réglette commandant le cylindre soit à son tour perpendiculaire au cadre ; on évite ainsi l'accıochage de la première tige dans le couvain. L'index indique alors le nombre de points attribuer à la surface de couvain et qui sera additionné avec les points des autres cadres de la ruche.

b) Méthode d'utilisation à une personne. In conservant les positions de l'appareil décrites cidessus l'expérimentateur introduit la tige ronde dans une cellule d'un bord de l'ellipse et tire l'appareil jusqu'à l'autre extrémité (fig. 2), de même avec l'autre axe (fig. 3). Il peut arriver que les cellules se déchirent sous la poussée de la tige sui y est introduite; il serait donc préférable pour pratiquer ce cette méthode de construire l'appareil avec plusieurs tiges, qui en pénét rant dans autant de cellules répartiraient la poussée. Cette dernière disposition présente d'ailleurs fuelques inconvénients.

\section{RÉSUITATS. CONFRONTATION AVEC IES AUTRES MÉTHODES}

L'utilisation dans plusieurs expériences de l'appareil qui vient d'être décrit et la confrontation des résultats obtenus avec ceux qui sont fournis par d'autres méthodes nous permettent déjà certaines conclusions.

La précision, on le sait, est la principale des qualités requises pour un appareil de cette nature. Nous examinerons donc tout d'abord les résultats obtenus de ce point de vue. Dans une étude précédente nous avions mesuré 20 surfaces de couvain

TABLEAU 3

Comparaison entre les risultats obtenus par la mithode de mesure des aves de lellipse el ceux qu' l'on obtient ave l'appareil dítrit

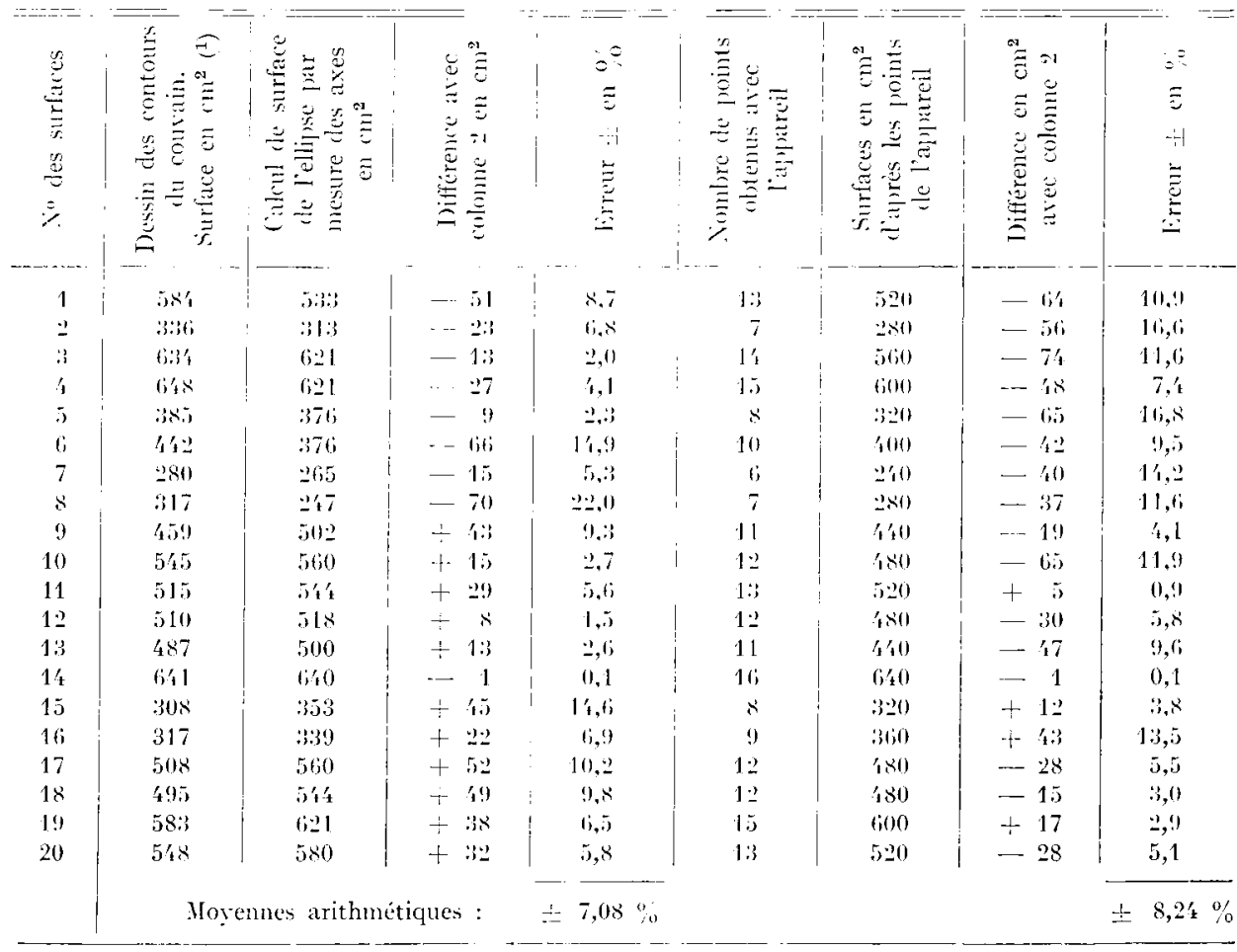

(1) D'apris Fresnate et Jlenskl, 1961. 
par la méthode du dessin des contours sur feuilles de cellophane (FRESNAYE et LENSKY I96I). J'ai donc opéré sur ces mêmes surfaces d'une part avec la méthode de mesure des axes de l'ellipse, d'autre part avec l'appareil qui vient d'être décrit. Le tableau 3 permet de confronter les résultats obtenus. L'erreur est de $\pm 7,08 \mathrm{p}$. Ioo pour la méthode de mesure des axes de l'ellipse et de $\pm 8,24 \mathrm{p}$. Ioo pour les mesures avec l'appareil. On voit que la différence de $\pm \mathrm{I}, \mathrm{I} 6 \mathrm{p}$. Ioo entre les deux méthodes est très faible et les résultats obtenus avec l'appareil peuvent pratiquement être assimilés à ceux obtenus par la méthode de mesure des axes de l'ellipse dontl'erreur est de \pm I3,39 p. IOO (FRESNAYE et LENSKY I96I). Ceci n'a rien de surprenant puisque les deux méthodes appliquent en fait le même principe.

La rapidité d'exécution est aussi une qualité très importante et c'est ici qu'apparait l'avantage déterminant de l'emploi de l'appareil sur les autres méthodes. Le temps nécessaire aux mesures et aux notations est le même pour les deux méthodes lorsque l'on opère à deux parsonnes; les gestes sont sensiblement les mêmes. Le gain de temps porte sur les calculs qui, sans appareil, nécessitent environ 30 secondes par surface à l'aide d'une machine à calculer électrique rapide. L'utilisation de l'appareil nous permat d'effectuer mesures et calculs sur 8 ruches à l'heure ce qui est très satisfaisant.

Contrairement à d'autres méthodes celle que nous avons ainsi mise au point ne nécessite pas le secouage des abeilles, cause de perturbations importantes. Les erreurs personnelles sont réduites. Les résultats sont utilisables immédiatement, un simple calcul mental permettant d'obtenir la surface du couvain en $\mathrm{cm}^{2}$ si on le désire.

\section{DISCUSSION}

L'utilisation de l'appareil que nous préconisons, principalement au cours d'expériences groupant de nombreuses ruches, permet de connaître rapidement la surface du couvain tout en évitant des calculs longs et fastidieux, le tout avec un pourcentage d'erreur voisin de celui qui est inhérent à la méthode de mesure des axes de l'ellipes ( \pm I3 p. Ioo environ). La facilité d'emploi et la rapidité d'exécution en font le procédé de mesure des surfaces de couvain le plus satisfaisant actuellement.

$$
\text { Reç pour publication en janvier } 1962 .
$$

\section{SUMMARY}

APPARATLS FOR EASY DETERMINATION OF TIE BROOD-COMB SURFACE IN BEEHIVES

Equipment which is easy to manipulate is described. It can give a rapid estimation of the size of brood-comb in beehives. This quick test can be used to determine the "brood-comb surface" criteria in experiments which include many beehives. It is essentially an aback by which the surface is directly determined from the coordinates engraved on two sliding rulers. The aback is rolled on a rotative cylinder. The precision of the technique is of about $\pm I 3 \%$ on the average, and it is sufficient for apicultural experiments.

\section{RÉFÉRENCE BIBLIOGRAPHIQUE}

Fresnaye J., Lensky Y., I96I. Méthodes d'appréciation des surfaces de couvain dans les colonies d'Abeilles Ann. Abeille, 4, 369-376. 